\title{
Evaluation of reservoir-induced hydrological alterations and ecological flow based on multi-indicators
}

\author{
Li Mingqian ${ }^{1}$, Liang Xiujuan ${ }^{1}$, Zhang Xuezhu ${ }^{2}$, Guiyang $\mathrm{Li}^{3}$, Hongying $\mathrm{Li}^{3}$, Wenhan \\ Jiang $^{4}$, and Changlai Xiao ${ }^{1}$ \\ ${ }^{1}$ Jilin University \\ ${ }^{2}$ Jilin Provincial Water Resources and Hydropower Consultative Company \\ ${ }^{3}$ Liaoning Provincial Department of Water Resources \\ ${ }^{4}$ Institute of Disaster Prevention Science and Technology
}

April 28, 2020

\begin{abstract}
Although they fulfill various needs of human beings, reservoirs also cause hydrological regime variation in the downstream regions, thus affecting ecological diversity. Therefore, studying the reservoir-induced hydrological alterations and ecological effects is of great significance, as it could guide the regulation of the reservoir to protect the river ecology. In this study, taking the Taizi River as an example, the impact of a reservoir on hydrological alteration and ecological diversity was comprehensively evaluated through eco-flow indicators based on the flow duration curve and multiple hydrological indicators. The results reveal that (1) the ecological indicators can be used to analyze the annual and seasonal changes in the streamflow after the construction of the reservoir. The high-flow values and frequency decrease after the construction of the reservoir, especially in the autumn, the low-flow component values increase significantly, especially in spring and summer; (2) the main influencing factors of the ecological indicators can be reflected by the relationship with precipitation, as the annual ecosurplus is not significantly affected by the reservoir, while the ecodeficit is greatly affected, and the seasonal ecological indicators (especially in spring and summer) are greatly affected by the reservoir; (3) the indicators of hydrologic alteration (IHA) show significant changes after the construction of the reservoir, and are consistent with the changes in the eco-flow indicators; the change in the Shannon Index indicates that the ecological diversity reduced after construction of the reservoir. It is controlled by the reservoir, and a new equilibrium state appears; and (4) the eco-flow indicators have a good correlation with the 32 IHAs; they can reflect the change information of most IHAs, and can avoid statistical redundancy.
\end{abstract}

\section{Hosted file}

Evaluation_of_reservoir-induced_hydrological_alterations_and_ecological_flow_based_on_multi-indicators. available at https : //authorea . com/users/286504/articles/410779-evaluation-of-reservoir-inducedhydrological-alterations-and-ecological-flow-based-on-multi-indicators 